\title{
Exercise Training Enhances Flow-Mediated Dilation in Spontaneously Hypertensive Rats
}

\author{
F. GÜNDÜZ ${ }^{1}$, G. KOÇER ${ }^{1}$, S. ÜLKER ${ }^{1}$, H. J. MEISELMAN ${ }^{2}$, O. K. BAŞKURT ${ }^{3}$, \\ Ü. K. ŞENTÜRK ${ }^{1}$
}

${ }^{1}$ Akdeniz University, Faculty of Medicine, Department of Physiology, Antalya, Turkey, ${ }^{2}$ University of Southern California, Keck School of Medicine, Department of Physiology and Biophysics, Los Angeles, CA, USA, ${ }^{3}$ Koc University School of Medicine, Rumelifeneri Yolu, Istanbul, Turkey

Received February 22, 2011

Accepted April 15, 2011

On-line May 16, 2011

\section{Summary}

This study investigated the effect of exercise training on the flowmediated dilation (FMD) in gastrocnemius muscle arteries from spontaneously hypertensive rats (SHR). SHR and WKY rats were divided into sedentary and exercised groups. After swimming exercise for eight weeks, the isolated arteries were mounted on pressurized myograph and FMD responses examined. The role of nitric oxide (NO), prostaglandins (PGs) and endothelium derived hyperpolarizing factor (EDHF) on FMD were assessed by obtaining dilation responses in the presence and absence of pharmacological antagonists. $\mathrm{N}^{\omega}$-nitro-L-arginine methyl ester (L-NAME), indomethacin (INDO) and tetraethylamonium (TEA) were used to inhibit nitric oxide synthase, cyclooxygenase and EDHF-mediated responses, respectively. The FMD response was significantly blunted in arteries of SHR compared with WKY rats, and, improved by exercise training in SHR (SHR-ET) group. In SHR arteries, L-NAME and TEA did not affect dilation responses to flow, while INDO led to a significant enhancement in this response. Although dilation response was not altered by L-NAME in arteries obtained from trained SHR, TEA caused a significant attenuation and INDO led to significant increases. These results demonstrate that exercise training improves FMD in SHR, and, this enhancement induced by exercise training occurs through EDHF-mediated mechanism(s).

\section{Key words}

EDHF • Nitric oxide $\bullet$ Prostaglandins

\section{Corresponding author}

F. Gündüz, Akdeniz University, Medical Faculty, Department of Physiology, Kampus, 07070, Antalya, TURKEY. Fax: +90 242 2274483. E-mail: fgunduz@akdeniz.edu.tr

\section{Introduction}

The endothelium plays an important role in the modulation of vascular tone through the production of numerous vasoactive substances. Several neurohumoral agents and mechanical forces such as shear stress that occur during blood flowing contribute to endotheliumdependent dilation (Mulvany and Aalkjaer 1990). Flowinduced shear stress regulates endothelial responses by modulating the release of endogenous factors such as nitric oxide (NO), prostaglandins (PGs) and results in flow-mediated dilation (FMD). On the other hand, endothelium-derived hyperpolarizing factor (EDHF) is another important mediator that is involved in FMD, thereby in endothelium-dependent dilation (Koller et al. 1994, Takamura et al. 1999). It is generally accepted that EDHF-mediated vasodilatory responses is associated with endothelial and smooth muscle cells hyperpolarization by opening potassium $\left(\mathrm{K}^{+}\right)$channels, without release any vasoactive factor (Nagao and Vanhoutte 1993).

Endothelial dysfunction that results from impaired endothelium-dependent dilation contributes to increased peripheral vascular resistance in hypertension (Folkow 1982, Priviero et al. 2009). Decreased relaxation response to both of neurohumoral factors and flow has been shown in many animal models of hypertension and humans (Koller and Huang 1994, Higashi and Yoshizumi 2004, Kuru et al. 2009, Priviero et al. 2009). Although the underlying mechanisms are controversial, several investigations have also demonstrated that vascular 
dilation response to increased flow is attenuated in small arteries responsible for peripheral vascular resistance in spontaneously hypertensive rats (SHR) that is an animal model of human essential hypertension (Koller and Huang 1994, Matrougui et al. 1997, Qiu et al. 1998, Koller and Huang 1999).

Exercise training improves endothelial function in hypertension (Chen et al. 1996, Higashi et al. 1999, Kuru et al. 2009). The most frequently proposed mechanism for this effect of exercise on endothelial function is the increased vascular blood flow and shear stress that stimulates endothelial nitric oxide synthase (eNOS)-dependent NO synthesis (Husain 2002, Higashi and Yoshizumi 2004). PGs and EDHF are other endothelium-dependent vasodilator factors that may participate in exercise training-induced vasodilation (Higashi and Yoshizumi 2004). On the other hand, the effect of regular physical exercise on endothelial function in SHR has been partly investigated. Several investigators have shown improved acetylcholine (ACh)-induced dilation responses in large and small artery segments obtained from exercised-trained SHR (Yen et al. 1995, Chen et al. 1996, 1999, Graham and Rush 2004). However, the possible influence of exercise on flowmediated dilation in SHR has not yet been clarified. The evaluation of FMD is important for the assessment of endothelial function, because of $\mathrm{ACh}$ and flow trigger endothelium-dependent dilation by different mechanisms.

The aim of the present study was to investigate how exercise training affects the FMD in small arteries in SHR. We hypothesized that attenuated FMD responses could be improved by exercise training in SHR. To test our hypothesis, we investigated the changes in dilation responses as a function of perfusate flow in isolated gastrocnemius muscle arteries of trained or untrained normotensive and hypertensive rats. In addition to assessing the possible role of NO, PGs and EDHFmediated responses in the modulation of FMD by exercise training, we also evaluated FMD response in the presence of pharmacological agents that block the synthesis and/or activities of those mediators.

\section{Methods}

Male spontaneously hypertensive rats (SHR) at 11-12 weeks of age and age-matched normotensive Wistar Kyoto (WKY) rats (Harlan Laboratories, USA) were used in the present study. The animals were housed at $23 \pm 2{ }^{\circ} \mathrm{C}$ on a $12: 12 \mathrm{~h}$ light-dark cycle and had free access to standard rat chow and drinking water. Rats were assigned randomly to four different groups: WKYsedentary (WKY, n=9), WKY-exercise training (WKYET, n=9), SHR-sedentary (SHR, n=8), and SHR-exercise training (SHR-ET, $n=9$ ). The animals in the exercise training groups were subjected to swimming exercise (60 min/day, five days/week for eight weeks) in a glass tank of $100 \times 50 \mathrm{~cm}$ with a depth of $50 \mathrm{~cm}$ filled with tap water $\left(32-34{ }^{\circ} \mathrm{C}\right)$. The duration of the first swimming experience was limited to $10 \mathrm{~min}$ and increased by $10 \mathrm{~min}$ daily until $60 \mathrm{~min}$ was reached. The experimental protocol was approved by the Animal Care and Usage Committee of Akdeniz University and was in accordance with the Declaration of Helsinki and International Association for the Study of Pain (IASP) guidelines.

The systolic blood pressure of all rats was measured using a non-invasive tail-cuff method at the beginning of the study (basal) and every two weeks during the eight week period. Data were obtained with a MAY-BPHR 9610-PC unit and MP 150 data acquisition system (BIOPAC Systems; Santa Barbara, CA-USA). The final measurements were performed one day after the last swimming session in exercising animals.

\section{Isolation of feed arteries}

Rats were anesthetized with an intraperitoneal injection of thiopental sodium $(80 \mathrm{mg} / \mathrm{kg}$ body weight) one day after the last exercise period in the training groups. The gastrocnemius-soleus muscle group was removed and transferred to a dissecting dish filled with ice-cold physiological saline solution (PSS) containing (in $\mathrm{mM}$ ) $145.0 \mathrm{NaCl}, 4.7 \mathrm{KCl}, 2.0 \mathrm{CaCl}_{2}, 1.17 \mathrm{MgSO}_{4}$, $1.2 \mathrm{NaH}_{2} \mathrm{PO}_{4}, 5.0$ glucose, 2.0 pyruvate, 0.02 EDTA, and 25.0 MOPS, $\mathrm{pH}=7.4$.

The gastrocnemius feed arteries $(\sim 200 \mu \mathrm{m}$ in diameter) were carefully dissected free under a dissecting microscope (SZ61, OLYMPUS, Tokyo, Japan). The isolated arterial segments were transferred to a vessel chamber (CH/1, Living Systems, Inc., Burlington, VT, USA) containing two horizontal glass micropipettes filled with PSS-albumin $(1 \mathrm{~g} / 100 \mathrm{ml})$. After the vessel was mounted on the proximal pipette and secured with 11-0 surgical nylon suture the perfusion pressure was raised to $20 \mathrm{~mm} \mathrm{Hg}$ to clear clotted blood from the lumen. Then the other end of the vessel was mounted on the distal pipette. A pressure servo-controlled roller pump perfusion system (Living Systems, Inc., Burlington, VT) was connected to the proximal pipette, and a similar but manually-controlled roller pump attached to the distal 
end. The pressure in each pipette was monitored by pressure transducers, and thus the intraluminal pressure in the vessel could be controlled by the pressure servocontrolled perfusion system.

The mounted vessel segments were visualized by an inverted microscope (Eclipse TS100, Nikon) equipped with a charge-coupled device camera (XC73CE, Sony). The camera was connected to a video dimension-analysis system (model V94, Living Systems) which allowed continuous measurement of vessel diameter. Mean intraluminal pressure, pressure gradient, fluid flow rate through the arterial segment, and vessel diameter were continuously recorded via a data acquisition system (MP 100A-CE; BIOPAC Systems) connected to a personal computer.

The arteries were perfused with MOPS-PSS supplemented with albumin $(1 \mathrm{~g} / 100 \mathrm{ml})$ and the axial length of the arterial segment was adjusted by positioning the cannula until the vascular walls were parallel without obvious stretch. Vessels that were free from leaks were pressurized to $60 \mathrm{~mm} \mathrm{Hg}$ with the servo-controlled pump, gradually warmed to $37{ }^{\circ} \mathrm{C}$, and allowed to develop spontaneous tone during equilibration period. The preparations were left to equilibrate for one hour while the bathing solution was changed every $15 \mathrm{~min}$.

\section{Evaluation of flow mediated dilation responses}

Flow mediated dilation (FMD) responses were assessed using various flow rates between 7-45 $\mu \mathrm{l} / \mathrm{min}$ while keeping intraluminal pressure constant at $60 \mathrm{~mm}$ $\mathrm{Hg}$ by the pressure servo-controlled system. Each flow rate was maintained for five min to obtain a steady-state vessel diameter. After obtaining control responses the arterial segment was washed and the role of NO in the mediation of FMD responses was assessed. The relative contribution of NO was evaluated by examining FMD responses in the presence of $\mathrm{N}^{\omega}$-nitro-L-arginine methyl ester (L-NAME, $10^{-4} \mathrm{M}$ ), an inhibitor of NOS. Vessels were incubated with L-NAME for $20 \mathrm{~min}$ and than FMD responses were reassessed. The role of prostaglandins was assessed after $20 \mathrm{~min}$ incubation period with indomethacin (INDO, $10^{-5} \mathrm{M}$ ), an inhibitor of cyclooxygenase (COX). Finally, after washing the vessel, a $\mathrm{K}^{+}$channel blocker, tetraethylamonium (TEA, $10^{-3} \mathrm{M}$ ) was added in the bath solution, and, after $20 \mathrm{~min}$ incubation period dilation responses were reevaluated to determine the role of non-NOS and non-COX pathways in the FMD.

At the end of experiment, the MOPS-PSS bath solution was replaced with $\mathrm{Ca}^{2+}$-free PSS and the vessels were incubated at least for $30 \mathrm{~min}$ to determine their maximal passive diameter.

\section{Statistics}

All values are given as means \pm S.E.M. Changes in diameter in response to increases in the perfusate flow were normalized to the corresponding passive diameter and expressed as percent maximal response by using following calculation: $\left(D_{d}-D_{b}\right) /\left(D_{p}-D_{b}\right) \times 100$ where $D_{d}$ is the measured diameter for a given flow; $D_{b}$ is the baseline diameter before an intervention was started, and $\mathrm{D}_{\mathrm{p}}$ is the maximal passive diameter. Initial tone is expressed as a percentage of maximal passive diameters. Between-group differences in blood pressure, maximal passive diameter and the initial tone of vessels from WKY and SHR rats were assessed using one-way ANOVA. Two-way ANOVA with repeated measures was used for comparison of the flow-dilation response curves and blood pressure levels; the Bonferroni test was used as a post-hoc test. $\mathrm{P}<0.05$ values were considered to be significant.

Table 1. Systolic blood pressure levels in normotensive and hypertensive rats during the eight week period.

\begin{tabular}{|c|c|c|c|c|c|}
\hline & \multicolumn{5}{|c|}{ Weeks } \\
\hline & 0 & 2 & 4 & 6 & 8 \\
\hline & \multicolumn{5}{|l|}{$m m ~ H g$} \\
\hline$W K Y$ & $129.8 \pm 1.1$ & $130.6 \pm 1.4$ & $130.3 \pm 1.6$ & $131.1 \pm 1.1$ & $133.8 \pm 1.1$ \\
\hline$W K Y-E T$ & $131.2 \pm 1.0$ & $130.7 \pm 1.1$ & $131.6 \pm 1.2$ & $130.0 \pm 1.5$ & $133.1 \pm 1.2$ \\
\hline SHR & $190.8 \pm 1.3^{*}$ & $191.9 \pm 1.0 *$ & $195.8 \pm 1.4^{*}$ & $199.1 \pm 1.2 *$ & $203.9 \pm 1.2 *$ \\
\hline$S H R-E T$ & $192.0 \pm 1.7^{*}$ & $188.8 \pm 1.2^{*}$ & $188.4 \pm 0.9^{* \dagger}$ & $190.4 \pm 1.0 * \dagger$ & $183.7 \pm 2.0 * \dagger$ \\
\hline
\end{tabular}

Values are given as the mean \pm S.E.M. ${ }^{*} p<0.001$ difference from $W K Y, t p<0.001$ difference from SHR. WKY, Wistar Kyoto; SHR, spontaneous hypertensive rat; WKY-ET, Wistar Kyoto exercise-trained; SHR-ET spontaneous hypertensive rat exercise-trained. 


\section{Results}

Systolic blood pressure (SBP) levels were elevated in SHR compared to WKY rats and exercise training induced a significant decrease in blood pressure in SHR compared to the untrained-SHR group (Table 1). The difference became significant at the $4^{\text {th }}$ week of exercise and continued untill the end of experiment. There was no significant change in SBP in exercisetrained normotensive rats (WKY-ET). Maximal passive diameters and initial tone of gastrocnemius arteries were similar in all groups (data not shown).

Vasodilation responses to flow and effect of exercise training

Vasodilation in response to intraluminal flow was decreased in gastrocnemius arteries from SHR compared to those from WKY rats. Exercise resulted in a significant improvement in dilation response to flow in SHR but not in WKY rats (Fig. 1).

\section{Effect of NOS inhibition}

NOS inhibition with L-NAME diminished FMD in gastrocnemius arteries for both WKY and WKY-ET rats (Fig. 2A and B). However, dilation responses to flow were not altered by NOS inhibition with L-NAME in the SHR and SHR-ET groups (Fig. 2C and D).

\section{Effect of COX inhibition}

INDO significantly reduced FMD in gastrocnemius arteries of both untrained and trained normotensive rats (Fig. $3 \mathrm{~A}$ and B). However, in hypertensive rats, COX inhibition by INDO caused significant increases in FMD, mainly for the nonexercised SHR rats (Fig. 3C and D).

\section{Effect of potassium channel inhibition}

Dilation responses to increase in perfusate flow in arteries from WKY and WKY-ET rats were significantly decreased after $\mathrm{K}^{+}$channel inhibition with TEA (Fig. 4A and B). TEA did not alter FMD in arteries of the SHR group (Fig. 4C) whereas it resulted in significantly decreased dilation responses in arteries obtained from SHR-ET (Fig. 4 D).

\section{Discussion}

The aims of present study were: 1) to determine whether exercise training restores the attenuated FMD

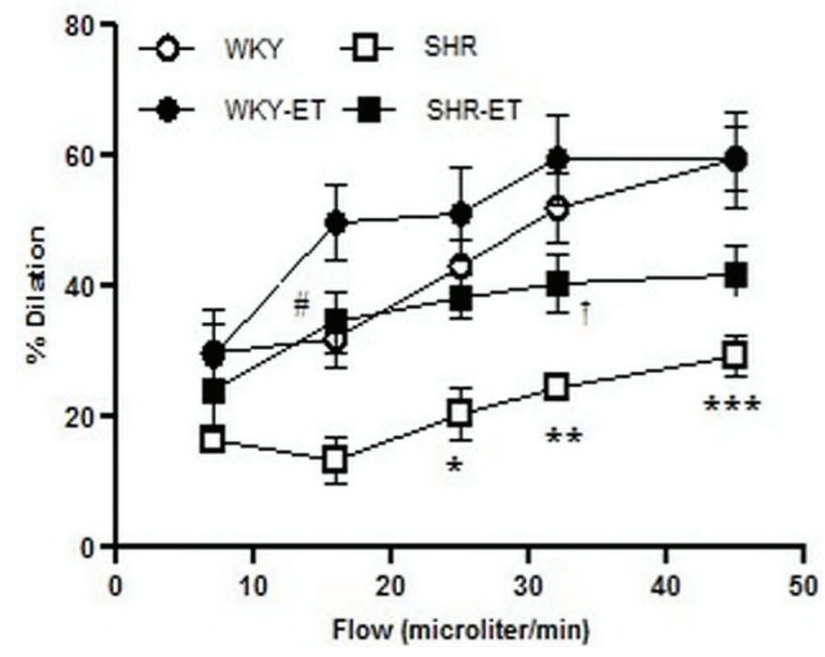

Fig. 1. Flow-mediated dilation (FMD) response in gastrocnemius feed arteries from normotensive (WKY) and hypertensive (SHR) rats and the effect of exercise on FMD. Values are means \pm S.E.M. ${ }^{*} p<0.05, * * p<0.01, * * * p<0.001$, difference from WKY; ${ }^{\#} \mathrm{p}<0.05$, difference from SHR, ${ }^{+} \mathrm{p}<0.05$, difference from WKY-ET.

response in SHR; 2) to investigate possible mechanism or mechanisms that mediate an effect of exercise training. Our results clearly demonstrate that exercise training countered the reduction of FMD in gastrocnemius muscle arteries from SHR, and that this improvement appears to be linked to EDHF-related mechanism(s).

It is known that altered behavior of vascular endothelial cells, as well as morphological changes of vascular wall, is involved in the development of hypertension (Folkow 1982, Mulvany 1993). Endothelial dysfunction has been well defined in SHR which is an animal model simulating human essential hypertension (Félétou et al. 2009). Reduced NO bioavailability, altered PGs production and/or efficacies are most proposed mechanisms that contribute to endothelial dysfunction and endothelium-dependent contraction that is elicited by ACh in SHR aortas (Félétou et al. 2009). On the other hand, a marked decrease of EDHF-mediated responses has been shown in resistance arteries obtained from SHR (Mantelli et al. 1995, Mori et al. 2006). Thereby, the endothelial dysfunction observed in small arteries in SHR seems to be related with diminished EDHF-mediated mechanism(s).

It has been suggested that the beneficial effect of exercise on endothelial function involves the blood pressure lowering effect of exercise (Higashi et al. 1999, Husain 2002, Higashi and Yoshizumi 2004, Kuru et al. 2009). The improvement of vascular dilation response to $\mathrm{ACh}$ in SHR has also been presented by previous studies 

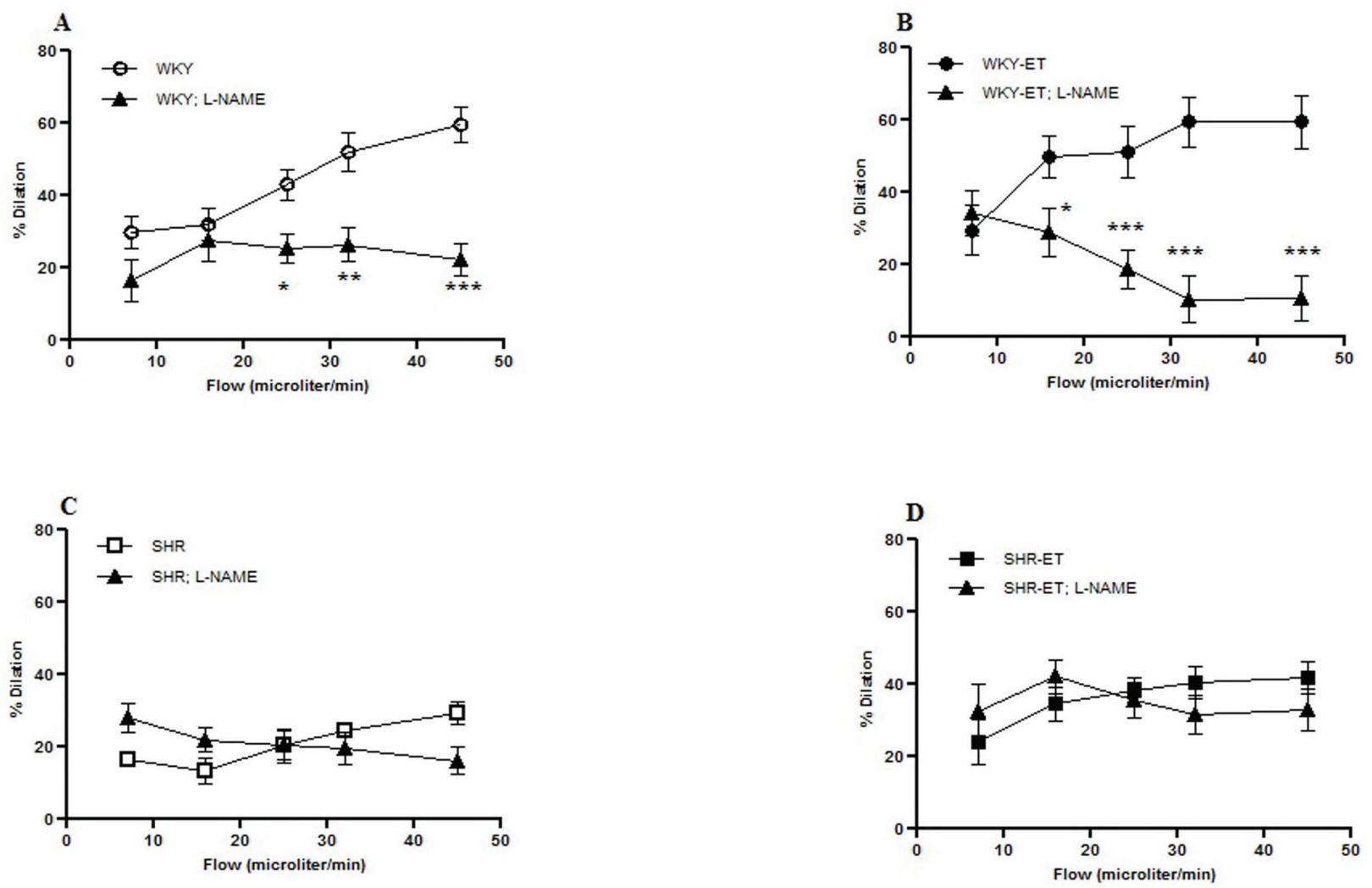

Fig. 2. Effect of nitric oxide synthase (NOS) inhibition on flow-mediated dilation (FMD) response in gastrocnemius feed arteries. A and B: Effect of NOS inhibition on FMD response in WKY and WKY-ET. C and D: Effect of NOS inhibition on FMD response in SHR and SHR-ET. Values are means \pm S.E.M. ${ }^{*} \mathrm{p}<0.05,{ }^{* *} \mathrm{p}<0.01, * * * \mathrm{p}<0.001$, difference from WKY or WKY-ET.
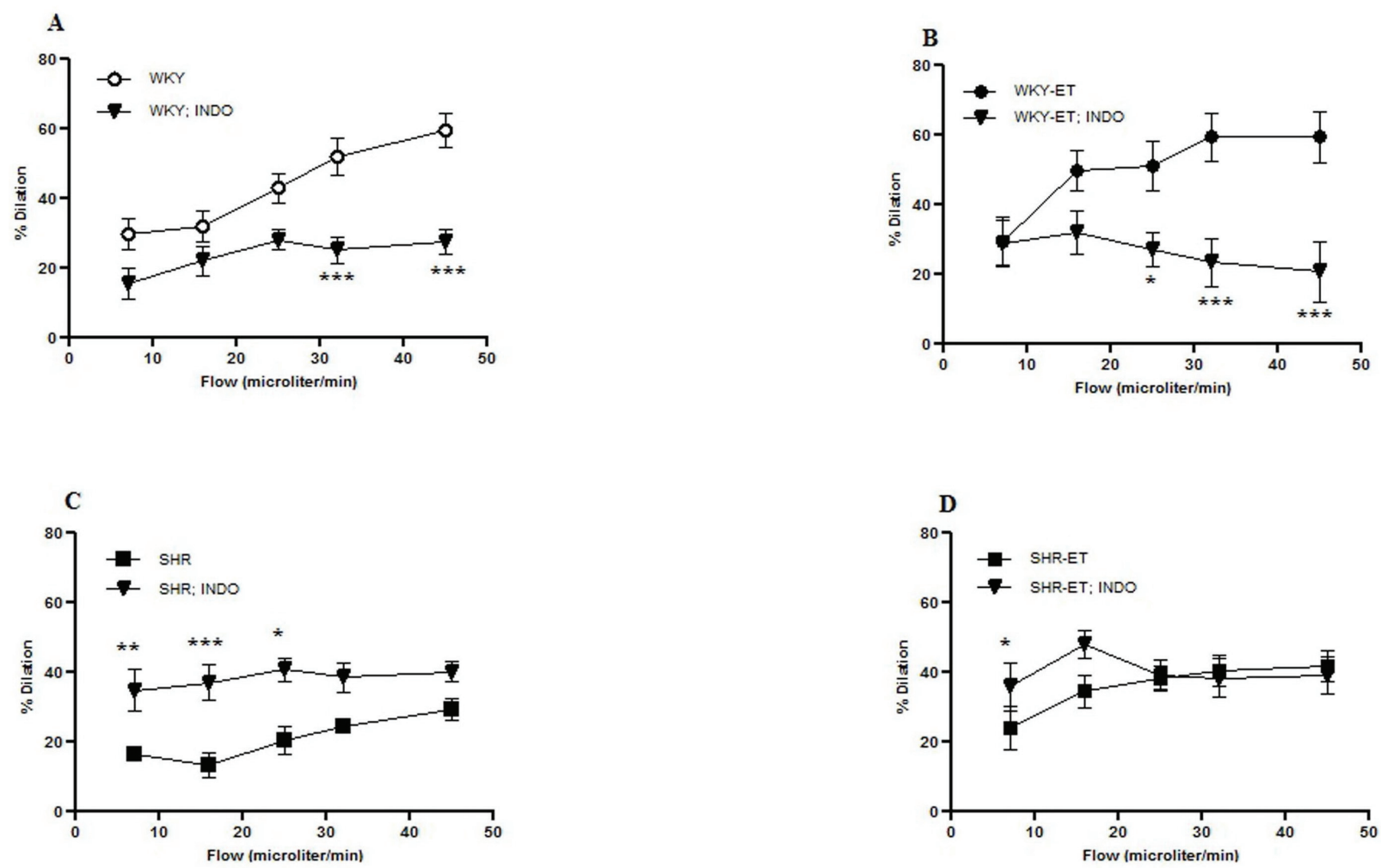

Fig. 3. Effect of cyclooxygenase (COX) inhibition on flow-mediated dilation (FMD) response in gastrocnemius feed arteries. A and $\mathbf{B}$ : Effect of COX inhibition on FMD response in WKY and WKY-ET. C and D: Effect of COX inhibition on FMD response in SHR and SHR-ET. Values are means \pm S.E.M. ${ }^{*} p<0.05, * * p<0.01, * * * p<0.001$, difference from WKY or WKY-ET, SHR or SHR-ET. 
(Yen et al. 1995, Chen et al. 1996). In our present investigation, we observed that swimming training caused a decrease of blood pressure in hypertensive rats beginning from the $4^{\text {th }}$ week of exercise until the end of the experiment (Table1). Lowering blood pressure by physical exercise has been demonstrated in hypertensive rats by several investigators (Yen et al. 1995, Kuru et al. 2002, Horta et al. 2005, Bertagnolli et al. 2008), although some studies did not confirm these findings (Graham and Rush 2004). These discrepancies may be explained by the different exercise (intensity, duration or kind) or age of animals used in those studies.

Impaired dilation response to flow has also been previously demonstrated in SHR small arteries (Koller and Huang 1994, Matrougui et al. 1997, Qiu et al. 1998, Koller and Huang 1999), and our results are consistent with these reports (Fig. 1). On the other hand, it is not clear whether regularly physical activity affects the responses of small arteries to flow in SHR. To our knowledge, this is the first study investigating the possible exercise training-induced alterations in response to flow of resistance arteries from trained SHR. The primary finding of this study is the exercise training improves the FMD responses in gastrocnemius arteries from SHR (Fig. 1). Additionally, the possible role of NOS, COX and/or EDHF related mechanisms in exercise training-induced improvement in FMD responses was also evaluated in the present study.

The role of NO production in the response to flow is well known (Koller et al. 1994). Flow-induced NO production is the most frequently proposed mechanism for the beneficial effect of exercise training on endothelial function (Higashi et al. 1999, Higashi and Yoshizumi 2004). In agreement with these observations, we also determined a significant decrease in FMD response after L-NAME treatment in both of WKY and WKY-ET rats (Fig. 2A and B). However, the alreadyreduced dilation response to flow of SHR arteries was not further decreased after L-NAME incubation period (Fig. 2C). The dilation response to flow was elevated by exercise training in SHR-ET, but this response was not affected by NOS inhibition (Fig. 2D). Our results suggest that NO does not play an important role in FMD in genetically hypertensive rats.

The NO pathway is altered in hypertension. Prior studies have shown that the attenuated dilation response to flow in small arteries from SHR is insensitive to NO synthesis blockade (Koller and Huang 1994, Matrougui et al. 1997, Qiu et al. 1998, Koller and Huang
1999). Although the mechanism underlying the attenuation is not yet clear, it has been proposed that the signal transduction, which links flow or shear stress to NO release, might be altered in SHR (Koller and Huang 1999). However, Qiu et al. (1998) demonstrated that although flow-induced NOS activity and cGMP release were significantly greater in mesenteric resistance arteries of SHR than in those of WKY, the dilation response to flow was markedly decreased in the SHR arteries. This decreased dilation response from SHR despite increased flow-induced cGMP production supports the idea that cGMP might be less efficient in these animals. On the other hand, decreased NO bioavailability might be involved in endothelial dysfunction in hypertension (Priviero et al. 2009). Some studies suggest that increased superoxide anion and NO production in hypertension which may result in peroxynitrite formation and decreased NO bioavailability (Grunfeld et al. 1995, Pecháňová and Šimko 2007).

Presumably, the participation of PGs in the dilation response to flow is variable depending on the anatomic localization of the vascular bed and/or the species (Friebel et al. 1995, Matrougui et al. 1997), similar to NO. Involvement of PGs in the dilation response was investigated using indomethacin, and our results demonstrated that PGs-induced dilation is a part of the FMD response from trained and untrained WKY rats (Fig. 3A and B). This finding is in agreement with results obtained from rat skeletal muscle arterioles (Koller et al. 1993, Friebel et al. 1995). On the other hand, whether the flow-induced dilation is sensitive to PGs synthesis blockade in small arteries in SHR is still controversial. Koller and Huang (1994) showed that dilation response to flow decreased during PG synthesis blockade in gracilis muscle arterioles, whereas COX blockade did not affect this response in mesenteric resistance arteries of SHR (Matrougui et al. 1997).

Interestingly, our results demonstrated that the dilation response to flow in muscle resistance arteries in SHR was augmented by PGs synthesis blockade (Fig. 3C). This finding correlates with an impaired AChinduced response being restored in SHR aorta and small mesenteric arteries by COX inhibition (Lüscher et al. 1990, Graham and Rush 2009). Although we could not evaluate vascular COX expression in the present study (due to the inadequate specimen), several previous studies have shown increased vascular production of constrictor PGs and increased vascular COX-1 expression in SHR (Ge et al. 1995, Huang et al. 2000), and, that 

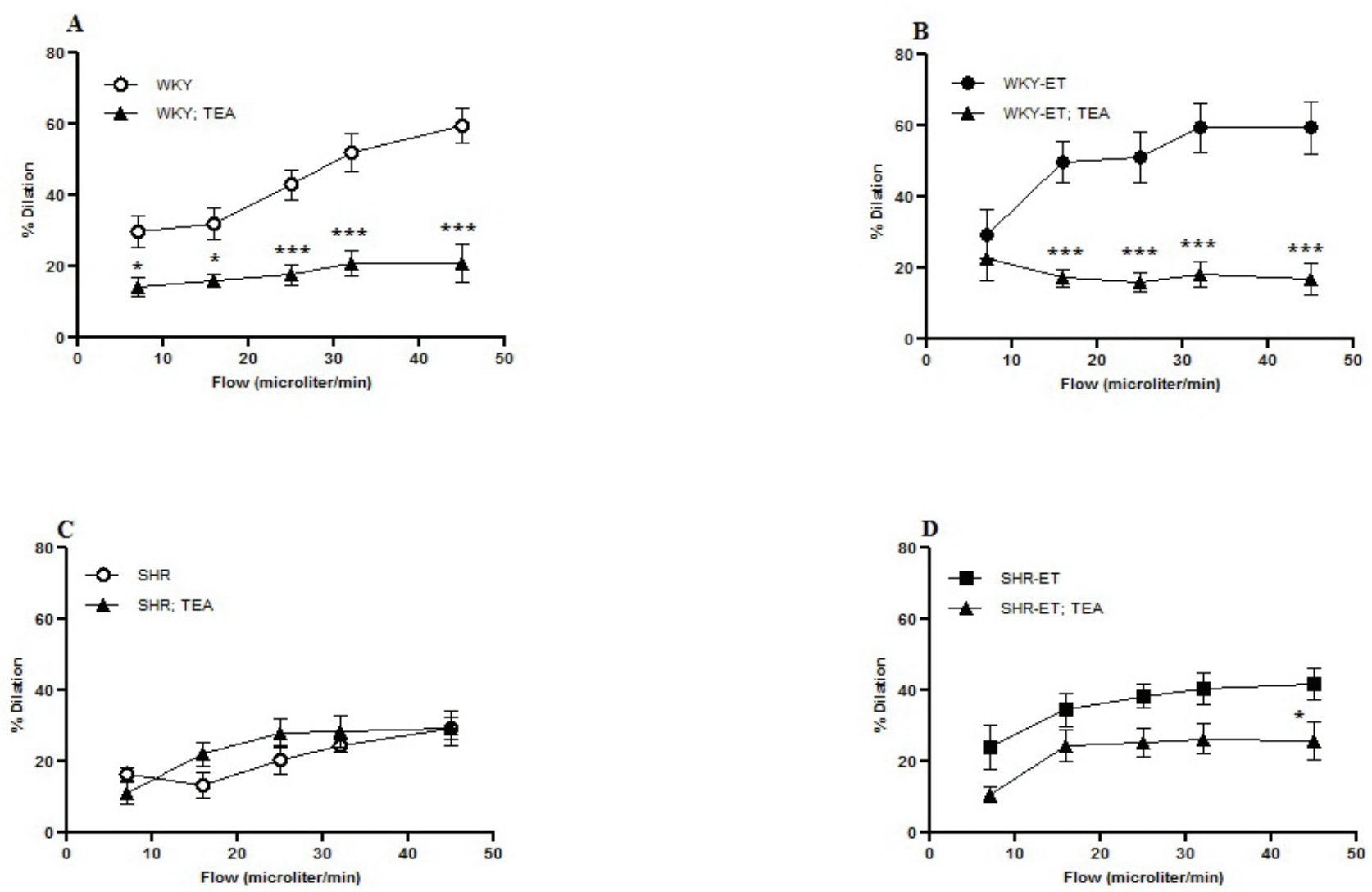

Fig. 4. Effect of endothelium-derived hyperpolarizing factor (EDHF) inhibition on flow-mediated dilation (FMD) response in gastrocnemius feed arteries. A and B: Effect of EDHF inhibition on FMD response in WKY and WKY-ET. C and D: Effect of EDHF inhibition on FMD response in SHR and SHR-ET. Values are means \pm S.E.M. ${ }^{*} p<0.05, * * p<0.01, * * * p<0.001$, difference from WKY or WKY-ET or SHR-ET.

these changes progress with age (Tang and Vanhoutte 2008, Graham and Rush 2009). Moreover, endothelial impairment as well as increases in blood pressure become more evident with age in genetically hypertensive rats (Koller and Huang 1999). The above mentioned studies (Koller and Huang 1994, Matrougui et al. 1997) were performed in 12-week-old SHR whereas our animals were about 20 weeks old at the time of the experiment because of accomplishing the eight-week training period. When all these findings are taken together, it could be speculated that a possible age-related alteration(s) in PGs production might contribute to the endothelial dysfunction that is evident with aging of SHR. FMD response in the presence of INDO displayed a small but significant increment in trained SHR group (Fig 3D). Thus our result suggests that PGs might not be involved in exercise-induced improvement of FMD in SHR.

EDHF-related mechanism(s) is one of the important mediators involved in endothelium-dependent vasodilation. It hyperpolarizes vascular smooth muscle cells by opening potassium channels (Nagao and Vanhoutte 1993, Shimokawa et al. 1996). It has been shown that EDHF-mediated vascular relaxation represents a part of the flow- or shear stress-induced dilation, and that it has physiological importance in both small and large arteries (Shimokawa et al. 1996). Although the role of $\mathrm{NO}$ and PGs in flow-induced dilation in small arteries from SHR has been widely investigated (Holtz et al. 1984, Koller et al. 1993, Friebel et al. 1995), the role of EDHF remains to be elucidated.

In this present study, we used TEA, a nonselective potassium channel inhibitor, to investigate whether EDHF pathway contributes to the FMD response in our experimental groups. Our results demonstrated that TEA caused a significant reduction in response to flow in WKY and WKY-ET rats (Fig. 4A and B) and are in agreement with results obtained in mesenteric small arteries of normotensive rats (Takamura et al. 1999). On the other hand, the FMD response was not influenced by potassium channel blockade in SHR whereas it was significantly reduced at $45 \mu \mathrm{l} / \mathrm{min}$ flow rate in SHR-ET (Fig. 4C and D). This observation suggests that the EDHF pathway may also be altered in hypertensive rats and involved in the beneficial effect of exercise training 
on dilation response to flow in SHR. Although this is the first study, which demonstrates the role of EDHF in exercise-induced flow-mediated vasodilation in SHR, it has been shown that EDHF-related mechanism(s) contributes to ACh-induced dilation in mesenteric arteries from exercised SHR (Yen et al. 1995).

In summary, the results of this study indicate that dilation response to flow was improved by regular exercise training in gastrocnemius arteries of SHR. This improvement effect of exercise training seems to be by means of EDHF-related mechanism(s).

\section{Conflict of Interest}

There is no conflict of interest.

\section{Acknowledgements}

This study was supported by Akdeniz University Research Projects Unit (Project Number 2006.01.0103.011) and by NIH Research Grants HL15722 and HL 70595.

\section{References}

BERTAGNOLLI M, SCHENKEL PC, CAMPOS C, MOSTARDA CT, CASARINI DE, BELLÓ-KLEIN A, IRIGOYEN MC, RIGATTO K: Exercise training reduces sympathetic modulation on cardiovascular system and cardiac oxidative stress in spontaneously hypertensive rats. Am J Hypertens 21: 1188-1193, 2008.

CHEN HI, CHIANG IP, JEN CJ: Exercise training increases acetylcholine-stimulated endothelium-derived nitric oxide release in spontaneously hypertensive rats. J Biomed Sci 3: 454-460, 1996.

CHEN Y, COLLINS, HL, DICARLO SE: Daily exercise enhances acetylcholine-induced dilation in mesenteric and hindlimb vasculature of hypertensive rats. Clin Exp Hypertens 21: 353-376, 1999.

FÉLÉTOU M, VERBEUREN TJ, VANHOUTTE PM: Endothelium-dependent contractions in SHR: a tale of prostanoid TP and IP receptors. Br J Pharmacol 156: 563-574, 2009.

FOLKOW B: Physiological aspects of primary hypertension. Physiol Rev 62: 347-504, 1982.

FRIEBEL M, KLOTZ KF, LEY K, GAEHTGENS P, PRIES AR: Flow-dependent regulation of arteriolar diameter in rat skeletal muscle in situ: role of endothelium-derived relaxing factor and prostanoids. $J$ Physiol 483: 715726, 1995.

GE T, HUGHES H, JUNQUERO DC, WU KK, VANHOUTTE PM, BOULANGER CM: Endothelium-dependent contractions are associated with both augmented expression of prostaglandin $\mathrm{H}$ synthase-1 and hypersensitivity to prostaglandin H2 in the SHR aorta. Circ Res 76: 1003-1010, 1995.

GRAHAM DA, RUSH JW: Exercise training improves aortic endothelium-dependent vasorelaxation and determinants of nitric oxide bioavailability in spontaneously hypertensive rats. J Appl Physiol 96: 2088-96, 2004.

GRAHAM DA, RUSH JW: Cyclooxygenase and thromboxane/prostaglandin receptor contribute to aortic endotheliumdependent dysfunction in aging female spontaneously hypertensive rats. $J$ Appl Physiol 107: 1059-1067, 2009.

GRUNFELD S, HAMILTON CA, MESAROS S, MCLAIN SW, DOMINICZAK AF, BOHR DF, MALINSKI T: Role of superoxide in the depressed nitric oxide production by the endothelium of genetically hypertensive rats. Hypertension 26: 854-857, 1995.

HIGASHI, Y, SASAKI S, KURISU S, YOSHIMIZU A, SASAKI N, MATSUURA H, KAJIYAMA G, OSHIMA T: Regular aerobic exercise augments endothelium-dependent vascular relaxation in normotensive as well as hypertensive subjects: role of endothelium-derived nitric oxide. Circulation 100: 1194-1202, 1999.

HIGASHI Y, YOSHIZUMI M: Exercise and endothelial function: role of endothelium-derived nitric oxide and oxidative stress in healthy subjects and hypertensive patients. Pharmacol Ther 102: 87-96, 2004.

HOLTZ J, FÖRSTERMANN U, POHL U, GIESLER M, BASSENGE E: Flow-dependent, endothelium-mediated dilation of epicardial coronary arteries in conscious dogs: effects of cyclooxygenase inhibition. $J$ Cardiovasc Pharmacol 6: 1161-1169, 1984.

HORTA PP, DE CARVALHO JJ, MANDARIM-DE-LACERDA CA: Exercise training attenuates blood pressure elevation and adverse remodeling in the aorta of spontaneously hypertensive rats. Life Sci 77: 3336-3343, 2005.

HUANG A, SUN D, KOLLER A: Shear stress-induced release of prostaglandin H(2) in arterioles of hypertensive rats. Hypertension 35: 925-930, 2000. 
HUSAIN K: Exercise conditioning attenuates the hypertensive effects of nitric oxide synthase inhibitor in rat. Mol Cell Biochem 231: 129-137, 2002.

KOLLER A, HUANG A: Impaired nitric oxide-mediated flow-induced dilation in arterioles of spontaneously hypertensive rats. Circ Res 74: 416-421, 1994.

KOLLER A, HUANG A: Development of nitric oxide and prostaglandin mediation of shear stress-induced arteriolar dilation with aging and hypertension. Hypertension 34: 1073-1079, 1999.

KOLLER A, SUN D, KALEY G: Role of shear stress and endothelial prostaglandins in flow- and viscosity-induced dilation of arterioles in vitro. Circ Res 72: 1276-1284, 1993.

KOLLER A, SUN D, HUANG A, KALEY G: Corelease of nitric oxide and prostaglandins mediates flow-dependent dilation of rat gracilis muscle arterioles. Am J Physiol 267: H326-H332, 1994.

KURU O, SENTURK UK, DEMIR N, YESILKAYA A, ERGULER G, ERKILIC M: Effect of exercise on blood pressure in rats with chronic NOS inhibition. Eur J Appl Physiol 87: 134-140, 2002.

KURU O, SENTÜRK UK, KOÇER G, OZDEM S, BAŞKURT OK, CETIN A, YEŞILKAYA A, GÜNDÜZ F: Effect of exercise training on resistance arteries in rats with chronic NOS inhibition. J Appl Physiol 107: 896-902, 2009.

LÜSCHER TF, AARHUS LL, VANHOUTTE PM: Indomethacin improves the impaired endothelium-dependent relaxations in small mesenteric arteries of the spontaneously hypertensive rat. Am J Hypertens 3: 55-58, 1990.

MANTELLI L, AMERINI S, LEDDA F: Roles of nitric oxide and endothelium-derived hyperpolarizing factor in vasorelaxant effect of acetylcholine as influenced by aging and hypertension. J Cardiovasc Pharmacol 25: 595-602, 1995.

MATROUGUI K, MACLOUF J, LÉVY BI, HENRION D: Impaired nitric oxide- and prostaglandin-mediated responses to flow in resistance arteries of hypertensive rats. Hypertension 30: 942-947, 1997.

MORI Y, OHYANAGI M, KOIDA S, UEDA A, ISHIKO K, IWASAKI T: Effects of endothelium-derived hyperpolarizing factor and nitric oxide on endothelial function in femoral resistance arteries of spontaneously hypertensive rats. Hypertens Res 29: 187-195, 2006.

MULVANY MJ: Resistance vessel structure and the pathogenesis of hypertension. J Hypertens 11: S7-S12, 1993.

MULVANY MJ, AALKJAER C: Structure and function of small arteries. Physiol Rev 70: 921-961, 1990.

NAGAO T, VANHOUTTE PM: Endothelium-derived hyperpolarizing factor and endothelium-dependent relaxations. Am J Respir Cell Mol Biol 8: 1-6, 1993.

PECHÁŇOVÁ O, ŠIMKO F: The role of nitric oxide in the maintenance of vasoactive balance. Physiol Res 56: S7S16, 2007.

PRIVIERO FB, ZEMSE SM, TEIXEIRA CE, WEBB RC: Oxidative stress impairs vasorelaxation induced by the soluble guanylyl cyclase activator BAY 41-2272 in spontaneously hypertensive rats. Am J Hypertens 22: 493499, 2009.

QIU HY, HENRION D, BENESSIANO J, HEYMES C, TOURNIER B, LEVY BI: Decreased flow-induced dilation and increased production of cGMP in spontaneously hypertensive rats. Hypertension 32: 1098-1103, 1998.

SHIMOKAWA H, YASUTAKE H, FUJII K, OWADA MK, NAKAIKE R, FUKUMOTO Y, TAKAYANAGI T, NAGAO T, EGASHIRA K, FUJISHIMA M, TAKESHITA A: The importance of the hyperpolarizing mechanism increases as the vessel size decreases in endothelium-dependent relaxations in rat mesenteric circulation. J Cardiovasc Pharmacol 28: 703-711, 1996.

TAKAMURA Y, SHIMOKAWA H, ZHAO H, IGARASHI H, EGASHIRA K, TAKESHITA A: Important role of endothelium-derived hyperpolarizing factor in shear stress-induced endothelium-dependent relaxations in the rat mesenteric artery. J Cardiovasc Pharmacol 34: 381-387, 1999.

TANG EH, VANHOUTTE PM: Gene expression changes of prostanoid synthases in endothelial cells and prostanoid receptors in vascular smooth muscle cells caused by aging and hypertension. Physiol Genomics 32: 409-418, 2008.

YEN MH, YANG JH, SHEU JR, LEE YM, DING YA: Chronic exercise enhances endothelium-mediated dilation in spontaneously hypertensive rats. Life Sci 57: 2205-2213, 1995. 